\title{
Examination of the FDA Warning for Statins and Cognitive Dysfunction
}

Frances M Sahebzamani ${ }^{*}{ }^{*}$, Cindy L Munro ${ }^{1}$, Oscar C Marroquin ${ }^{2}$, David M Diamond ${ }^{3,4}$, Erin Keller ${ }^{5}$ and Kevin E Kip ${ }^{1}$

${ }^{1}$ University of South Florida, College of Nursing, Tampa FL, USA

${ }^{2}$ University of Pittsburgh Medical Center, Division of Provider Analytics, Pittsburgh, PA, USA

${ }^{3}$ Research and Development Service, VA Hospital, Tampa, FL, USA

${ }^{4}$ Departments of Psychology and Molecular Pharmacology and Physiology, Center for Preclinical and Clinical Research on PTSD, University of South Florida, Tampa, FL, USA

${ }^{5}$ Denison University, Granville $\mathrm{OH}, \mathrm{USA}$

*Corresponding author: Frances M. Sahebzamani, Ph.D., Moffitt, University of South Florida, College of Nursing, 12901 Bruce B. Downs Blvd. MDN, Room 2010 Tampa, FL 33612-4476, USA, Tel: (813) 974-2702; Fax: (813) 974-7903; E-mail: fsahebza@health.usf.edu

Received date: June 18, 2014, Accepted date: August 26, 2014, Published date: September 02, 2014

Copyright: (c) 2014 Sahebzamani FM, et al. This is an open-access article distributed under the terms of the Creative Commons Attribution License, which permits unrestricted use, distribution, and reproduction in any medium, provided the original author and source are credited.

\begin{abstract}
Background: The ACC/AHA released new guidelines in December of 2013 for treatment of high blood cholesterol to simplify identification and treatment of patients most likely to benefit from statins. These guidelines may result in more patients receiving statin therapy, and at younger ages. In 2012, the U.S. Food and Drug Administration (FDA) mandated warnings for all statin drugs for possible adverse effects on cognitive performance. Statins can be classified as having greater lipophilic or hydrophilic solubility properties with lipophilic statins more readily crossing the blood brain barrier, and possibly differentially inducing detrimental cognitive effects.
\end{abstract}

Objective: We sought to analyze generalizability of the FDA statin class warning.

Methods: De-identified publicly-available data were analyzed from the FDA Adverse Event Reporting System (AERS) in relation to reports of cognitive dysfunction (primary outcome), and by type of statin (lipophilic, hydrophilic) versus "control" drugs used in the general population.

Results: Significantly higher proportional reporting ratios (PRRs) were observed for lipophilic statins, which more readily cross the blood-brain barrier, (range: 1.47-3.51) compared to hydrophilic statins (range: 0.69-1.64). However, fluvastatin, lovastatin, and pitavastatin (lipophilic) had relatively few adverse reports. The signal of higher risk of cognitive dysfunction was observed for the lipophilic statin atorvastatin (PRR $=2.59,95 \%$ confidence interval: 2.44-2.75) followed by simvastatin ( $P R R=2.22,95 \%$ confidence interval: 2.04-2.31). Hydrophilic statins (rosuvastatin, pravastatin) showed essentially no evidence suggestive of heightened risk of cognitive dysfunction. Fluvastatin, lovastatin, and pitavastatin had relatively few adverse reports, and no evidence of a higher proportion of cognitive dysfunction reports compared to the control drugs in aggregate (PRR range: 0.22 to 1.48 ).

Conclusions: Inconsistent with the FDA class warning, highly lipophilic statins with specific pharmacokinetic properties (atorvastatin, simvastatin) appear to confer a significantly greater risk of adverse cognitive effects compared to other lipophilic statins and those with hydrophilic solubility properties.

Keywords: Adverse events; Amnesia; Cholesterol; Cognitive dysfunction; Food and Drug Administration; HMG-CoA Reductase inhibitors; Hydrophilic; Lipophilic; Memory; Pharmacoepidemiology; Statins
Abbreviations
ACC: American College of Cardiology; AERS: Adverse Event Reporting System; AHA: American Heart Association; ASCII: American Standard Code for Information Interchange; FDA: Food and Drug Association; LDL: Low Density Lipoprotein; MedDRA: Medical Dictionary for Regulatory Activities; PRR: Proportional Reporting Ratios; SAS: Statistical Analysis Software

\section{Introduction}

Statins are widely prescribed in the United States for primary and secondary cardiovascular risk reduction and constitute an estimated $\$ 26$ billion dollar per year market for pharmaceutical manufacturers [1]. The numbers of patients receiving statin therapy is likely to increase in the coming years based on the dissemination in 2013 of the American College of Cardiology/American Heart Association's (ACC/ AHA) updated clinical guidelines for the assessment and management of blood cholesterol for primary and secondary prevention [2]. Although the new guidelines simplify clinical treatment strategies, they promote a broader risk assessment tool which may increase the numbers of patients between the ages of 20 - 79 eligible for statin use for primary prevention to an estimated 46 million Americans [3]. Statins are believed to reduce cardiovascular risk by lowering serum total cholesterol and low density lipoprotein (LDL) cholesterol, 
Page 2 of 9

although, a number of pleiotropic effects including reductions in inflammatory cytokines and plasminogen activator levels, improved endothelial function, anti-thrombotic effects, and plaque stabilization have been proposed as equally important vascular benefits [4-6]. Although statins are among the most widely prescribed medications, they are also among the most controversial. Clinical trials dating back to the 1990s have generated compelling evidence as to the effectiveness of statins for the reduction of cardiovascular morbidity and mortality in patients with established coronary artery disease (i.e. secondary prevention) [7-9]. While these trials provide evidence for secondary prevention of coronary heart disease, statins have been plagued by conflicting evidence of potential associations with higher all-cause mortality including cancer, trauma and suicide, as well as adverse physical, psychological, and cognitive effects [10-16]. More recently, controversies surrounding statins stem from a lack of consensus in the scientific community as to the benefits of statins for primary prevention in the absence of established coronary artery disease [17-20]. Despite these unresolved controversies, statins are increasingly endorsed and prescribed for daily, long-term use for primary prevention across a broader spectrum of low- to moderaterisk patients including among children through older adults.

In the practice setting, the risk/benefit considerations of advising statin use in low- to moderate-risk patients has been checked, at least in part, by considerations of the economic costs associated with longterm statin use in healthy, asymptomatic patients, as well as by concerns over the potential for adverse effects on liver function and muscle tissue. Commonly recognized adverse effects associated with statins have mainly focused on their well-known potential for induced myalgia and rarely, rhabdomyolysis. Although reports of adverse neurocognitive effects associated with statin use have been previously published in the scientific literature $[12,21]$ and in patient-based reporting systems [22], adverse neurocognitive effects have been less well recognized and possibly missed by clinicians [22] particularly in light of research suggesting that statins may confer neuroprotective benefits on neurovascular and neurodegenerative diseases including vascular and Alzheimer's dementia [23-27]. In contrast, a post marketing surveillance study unmasked a number of adverse effects of statins on cognitive performance and psychological symptoms including memory loss, amnesia, altered mood and irritability, sleep disruption and confusion such that in 2012, the Food and Drug Administration (FDA) mandated warnings on all statin labels [28]. Although this warning extends to all statins, previous research to examine statin-induced neurocognitive effects has provided clinically important insights against a class effect depending on the lipophilicity and resultant tissue selectivity of the statin [29-32]. Specifically, statins can be classified as having greater lipophilic or hydrophilic solubility properties, with lipophilic statins tending to achieve higher levels of exposure in non-hepatic tissues as lipophilic statins passively and nonselectively diffuse into both hepatocyte and non-hepatocyte tissue; hydrophilic statins rely largely on active transport into hepatocyte to exert their effects [33-38]. High hepatoselectivity is thought to translate into a reduced risk of adverse effects [38].

Accordingly, we sought to investigate the effect of type of statin (lipophilic versus hydrophilic) on reports of cognitive dysfunction using the FDA Adverse Event Reporting System (AERS), and to gauge the generalizability of the FDA mandated statin-at-large warning. To our knowledge, no previous studies have used the AERS database for this purpose and in comparison to a range of "control" drugs commonly used in the general population (i.e. to parallel statin use).

\section{Materials and Methods}

The FDA AERS is a computerized information database designed to support the FDA's post-marketing safety surveillance program for all approved drug and therapeutic biologic products. The FDA uses AERS to monitor for new adverse events and medication errors that might occur with these marketed products, in a post-marketing setting [39]. Of note, adverse events reported for particular medications do not list the primary indication for which the medication was used (e.g. specific disease condition and on-label versus off-label use, dosages). Adverse events in AERS are coded to Medical Dictionary for Regulatory Activities (MedDRA) terminology [40]. Importantly, reporting of adverse events from the point of care into AERS is voluntary (passive surveillance) in the U.S., with estimates of only $1 \%$ to $10 \%$ of all true adverse reactions being chronicled [41,42]. With this known underreporting and other limitations-including the fact that FDA does not require proof of a causal relationship between a product and event and that the incidence of adverse events cannot be calculated from AERS-this system is most appropriately used for "signal" identification for potential safety concerns which may provide the rationale for further evaluation, such as through the conduct of epidemiological studies $[43,44]$ or prospective research.

\section{Database utilization and search terms}

All quarterly data files from the FDA AERS website [39] from January 1, 2004 through September 30, 2012 were downloaded in ASCII text format and converted to SAS ${ }^{\circledast}$ databases using the SAS System ${ }^{\oplus}$. These data included the 7 separate quarterly data files publicly available (demographics, drug information, reaction information, outcome information, source of reporting, therapy dates, and manner of use) which were merged together using a common deidentified unique record number. Although the data are publicly available and de-identified, formal Institutional Review Board approval for analyses of these data was obtained from the University of South Florida, which verified compliance with Federal Exemption criteria outlined in 45CFR46.101(b).

\section{AERS definitions}

The AERS reports contain limited clinical information, with more detailed information on timing and nature of each reported event. Occupation: The occupation of the reporter of the event (physician, pharmacist, other health professional, lawyer, or consumer).

\section{Type of report}

"Expedited" (15-day) and "periodic" reports are submitted by manufacturers; "direct" reports are voluntarily submitted to the FDA by non-manufacturers.

\section{Reported role}

Classification for the drug's reported role in the event ("primary suspect drug", "secondary suspect drug", concomitant", "interacting"). Reports in AERS are per incident; thus, multiple reports can exist for a single individual.

\section{Statins}

To investigate adverse event reports for the most commonly prescribed statins, searches within AERS were conducted for 
individual drug and trade names as follows: atorvastatin (Lipitor, Torvast), fluvastatin (Lescol), lovastatin (Mevacor, Altocor, Altoprev), pitavastatin (Livalo, Pitava), pravastatin (Pravachol, Selektine, Lipostat), rosuvastatin (Crestor), and simvastatin (Zocor, Lipex). Based on established pharmacological properties, rosuvastatin and pravastatin were grouped and analyzed as hydrophilic; the remaining statins were classified as lipophilic [5,38,45] although, fluvastatin has been classified as a less lipophilic statin $[44,46,47]$. Table 1 provides a summary of the pharmacokinetic properties of the individual statins evaluated. Statin combination preparations were not selected for inclusion to avoid potential misclassification of exposure and adverse effect risk associated with the combined drugs.

\begin{tabular}{|c|c|c|c|c|c|c|}
\hline Drug Name & Solubility & $\begin{array}{l}\text { Half-life } \\
\text { (hrs.) }\end{array}$ & $\begin{array}{l}\text { Log partition } \\
\text { (octanol:water) }\end{array}$ & Absorption & Bioavailability & $\begin{array}{l}\text { Metabolic } \\
\text { pathway }\end{array}$ \\
\hline Atorvastatin & Lipophilic & $11-30$ & 1.11 & $30 \%$ & $12 \%$ & CYP3A4 \\
\hline Pitavastatin & Lipophilic & 11 & 1.49 & $80 \%$ & $>60 \%$ & CYP2C9 \\
\hline Lovastatin & Lipophilic & $2.5-3$ & 1.70 & $31 \%$ & $<5 \%$ & CYP3A4 \\
\hline Simvastatin & Lipophilic & $1.9-3$ & 1.60 & $65-85 \%$ & $<5 \%$ & CYP3A4 \\
\hline Fluvastatin & Lipophilic & $0.5-2.3$ & 1.27 & $98 \%$ & $10-35 \%$ & CYP2C9 \\
\hline Rosuvastatin & Hydrophilic & 20 & -0.33 & $50 \%$ & $20 \%$ & CYP2C9 \\
\hline Pravastatin & Hydrophilic & 11 & -0.84 & $37 \%$ & $18 \%$ & CYP3A4 \\
\hline
\end{tabular}

Table 1: Pharmacology of Individual Statins $[5,35,45,46,50]$.

\section{Control drugs}

We selected comparator drugs with similar consumption properties as statins, yet no known or suspected relationship with cognitive dysfunction. This included drugs with a high prevalence, potential daily use, different indications (e.g. pain, hypertension, gastroesophageal reflux disease), and frequent use among adults and older adults. The control drugs and their trade names were as follows: aspirin, ibuprofen, acetaminophen, diovan (Valsartan), enalapril (Renitec, Vasotec), hydrochlorothiazide, esomeprazole (Nexium), omeprazole (Losec, Omesec, Prilosec), clopidogrel (Plavix). For both statins and control drugs, adverse reports were only selected when the reported role in the adverse event was classified as "primary."

\section{Outcome reactions}

For determination of the primary outcome "cognitive dysfunction," two of the authors (F.S., K.K) reviewed a frequency listing of all names of adverse reports listed in AERS. By consensus, the following adverse reports were selected to comprise the broad category of cognitive dysfunction: amnesia (both unspecified type and global amnesia), cognitive disorder, confusional state, dementia, delusion, and memory impairment.

\section{Statistical analysis}

Adverse event reports for each individual statin and type of statin were considered the "signal" group and compared individually against the control drugs selected. Initially, chi-square tests were used to compare reporting characteristics of the statin and control drugs for reports in which cognitive dysfunction (as defined above) was listed as an adverse reaction. For all adverse event drug reports (statin and control), the proportion in which cognitive dysfunction was listed as an adverse reaction was calculated. These proportions formed the basis for calculating proportional reporting ratios (PRRs) with the statin drug (or type) in the numerator and control drug in the denominator. Thus, PRRs greater than one are indicative that the statin drug has a higher proportion of reported adverse events listed as cognitive dysfunction in comparison to the control drugs. When interpreting the PRR, prior reports (and in this analysis) of "signal" detection of heightened risk have used a minimum of three or more cases, $P R R \geq 2$, and chi-square value of $\geq 4.0$ [48]. $P$-values of the PRRs were calculated using chi-square tests with Yates correction and defining statistical significance as $P<0.05$.

\section{Results}

For the period January 1, 2004 through September 30, 2012, the "Drug" file within AERS contained a total of 16,471,747 records for evaluation. From these records, 1,270,721 adverse reports were identified for the collective set of statin and control drugs. Of these reports, 190,727 (15.0\%) were classified as being the primary suspected drug in the reaction and formed the basis of the analysis. The remaining adverse reports were classified as secondary suspect drug (11.2\%), concomitant drug (73.4\%), and interacting drug (0.3\%).

Of the 190,727 drug reports selected, 3,287 were for statins reported as the primary suspected drug related to cognitive dysfunction, and 1,580 were for the control drugs. The control drug reports for cognitive dysfunction were more likely than the statin reports to be among females (58.1\% versus $46.4 \%$ ) and submitted by consumers (52.2\% versus $29.0 \%)$. Of note, among lipophilic statin reports of cognitive dysfunction, $11.1 \%$ were submitted by a lawyer compared to just $0.5 \%$ among hydrophilic statin reports. For lipophilic statin reports of cognitive dysfunction, the percentages submitted by a lawyer over time were: 2001-2005 (1.4\%); 2006-2007 (18.8\%); 2008-2009 (21.8\%); 2010-2011 (10.0\%); 2012 (0.9\%).

\section{Proportional reporting of cognitive dysfunction}

Comparing the proportion of all adverse reports listed as cognitive dysfunction for statins versus the 8 individual control drugs, much higher PRRs were observed for lipophilic statins (range: 1.47 to 3.51 ) compared to hydrophilic statins (range: 0.69 to 1.64) [Table 2]. Six of the 8 PRR estimates for lipophilic statins versus individual control drugs exceeded the value of $>2$ specified as a signal of heightened risk 
Citation: Sahebzamani FM, Munro CL, Marroquin OC, Diamond DM, Keller E, et al. (2014) Examination of the FDA Warning for Statins and

Page 4 of 9

based on previous studies [48]. Results for hydrophilic statins showed essentially no signal suggestive of heightened risk of cognitive dysfunction, with a statistically lower prevalence reported in comparison to hydrochlorothiazide $(2.38 \%$ versus $3.46 \%$; $P R R=0.69)$. For the two hydrophilic statins and compared to all control drugs, rosuvastatin showed no indication of a higher proportion of cognitive dysfunction reports (PRR $=1.07,95 \%$ confidence interval: $0.97,1.25)$, whereas pravastatin suggested a nominally higher proportion $(\mathrm{PRR}=$ $1.90,95 \%$ confidence interval: $1.55,2.33$ ).

\begin{tabular}{|c|c|c|c|c|c|c|c|c|c|c|}
\hline Statin & $\mathbf{N}$ & Events & $\%$ & vs. Control & $\mathbf{N}$ & Events & $\%$ & PRR & $\begin{array}{l}95 \% \\
\text { C.I. }\end{array}$ & $P$-Value \\
\hline Lipophilic & 51,163 & 2,597 & 5.08 & Aspirin & 9815 & 142 & 1.45 & 3.51 & $\begin{array}{l}3.06 \\
4.34\end{array}$ & $<0.0001$ \\
\hline Lipophilic & 51,163 & 2,597 & 5.08 & Ibuprofen & 8,994 & 141 & 1.57 & 3.24 & $\begin{array}{l}2.73 \\
3.85\end{array}$ & $<0.0001$ \\
\hline Lipophilic & 51,163 & 2,597 & 5.08 & Acetaminophen & 11,017 & 196 & 1.78 & 2.85 & $\begin{array}{l}2.47 \\
3.30\end{array}$ & $<0.0001$ \\
\hline Lipophilic & 51,163 & 2,597 & 5.08 & Hydrochlorothiazide & 3,267 & 113 & 3.46 & 1.47 & $\begin{array}{l}1.22 \\
1.78\end{array}$ & $<0.0001$ \\
\hline Lipophilic & 51,163 & 2,597 & 5.08 & Diovan & 18,781 & 459 & 2.44 & 2.08 & $\begin{array}{l}1.88 \\
2.29\end{array}$ & $<0.0001$ \\
\hline Lipophilic & 51,163 & 2,597 & 5.08 & Enalapril & 2,464 & 72 & 2.92 & 1.74 & $\begin{array}{l}1.37 \\
2.21\end{array}$ & $<0.0001$ \\
\hline Lipophilic & 51,163 & 2,597 & 5.08 & Omeprazole & 8,133 & 200 & 2.46 & 2.06 & $\begin{array}{l}1.79 \\
2.39\end{array}$ & $<0.0001$ \\
\hline Lipophilic & 51,163 & 2,597 & 5.08 & Clopidogrel & 11,179 & 257 & 2.30 & 2.21 & $\begin{array}{l}1.94, \\
2.51\end{array}$ & $<0.0001$ \\
\hline Hydrophilic & 29,002 & 690 & 2.38 & Aspirin & 9815 & 142 & 1.45 & 1.64 & $\begin{array}{l}1.37 \\
1.98\end{array}$ & $<0.0001$ \\
\hline Hydrophilic & 29,002 & 690 & 2.38 & Ibuprofen & 8,994 & 141 & 1.57 & 1.52 & $\begin{array}{l}1.26, \\
1.82\end{array}$ & $<0.0001$ \\
\hline Hydrophilic & 29,002 & 690 & 2.38 & Acetaminophen & 11,017 & 196 & 1.78 & 1.34 & $\begin{array}{l}1.14 \\
1.57\end{array}$ & $<0.0001$ \\
\hline Hydrophilic & 29,002 & 690 & 2.38 & Hydrochlorothiazide & 3,267 & 113 & 3.46 & 0.69 & $\begin{array}{l}0.56 \\
0.84\end{array}$ & $<0.0001$ \\
\hline Hydrophilic & 29,002 & 690 & 2.38 & Diovan & 18,781 & 459 & 2.44 & 0.97 & $\begin{array}{l}0.86 \\
1.10\end{array}$ & 0.67 \\
\hline Hydrophilic & 29,002 & 690 & 2.38 & Enalapril & 2,464 & 72 & 2.92 & 0.81 & $\begin{array}{l}0.64, \\
1.04\end{array}$ & 0.11 \\
\hline Hydrophilic & 29,002 & 690 & 2.38 & Omeprazole & 8,133 & 200 & 2.46 & 0.97 & $\begin{array}{l}0.83, \\
1.13\end{array}$ & 0.70 \\
\hline Hydrophilic & 29,002 & 690 & 2.38 & Clopidogrel & 11,179 & 257 & 2.30 & 1.03 & $\begin{array}{l}0.90 \\
1.20\end{array}$ & 0.66 \\
\hline
\end{tabular}

Table 2: Proportional Reporting Ratios (PRR) of Statins to Control Drugs for Cognitive Dysfunction.

\section{Proportional reporting of individual lipophilic statins}

Given that the signal of cognitive dysfunction risk was restricted to the class of lipophilic statins, PRRs for individual lipophilic statins were calculated [Figure 1]. Fluvastatin, lovastatin, and pitavastatin had relatively few adverse reports in the AERS database [Figure 1, Panel $\mathrm{A}]$, and there was little to no evidence of a higher proportion of cognitive dysfunction reports compared to the control drugs in aggregate [PRR range: 0.22 to 1.48 , Figure 1 , Panel B]. In contrast, the signal suggestive of higher risk of cognitive dysfunction was observed for

\section{Proportional reporting of individual types of cognitive dysfunction}

In examining individual types of cognitive dysfunction reported, and restricting the analyses to atorvastatin and simvastatin (i.e. primary signals identified), the highest PRRs compared to all control drugs were for cognitive disorder (atorvastatin: 10.05; simvastatin: 6.31) followed by amnesia (atorvastatin: 8.12 ; simvastatin: 5.98 ) and then memory impairment (atorvastatin: 2.92; simvastatin: 2.32) [Table 3]. For all adverse events reported for atorvastatin, 3.2\% reported amnesia as a primary adverse reaction. Comparing atorvastatin versus 
simvastatin, the PRR was lower than the suggested signal detection value of 2.0 , yet statistically higher $(\mathrm{P}<0.0001)$ for reports of cognitive disorder ( $\mathrm{PRR}=1.59,95 \%$ confidence interval: $1.23,2.07)$ and amnesia
( $P R R=1.36,95 \%$ confidence interval: $1.20,1.54)$, while nominally higher for reports of memory impairment $(\mathrm{PRR}=1.26,95 \%$ confidence interval: $1.04,1.53, \mathrm{P}=0.02)$.
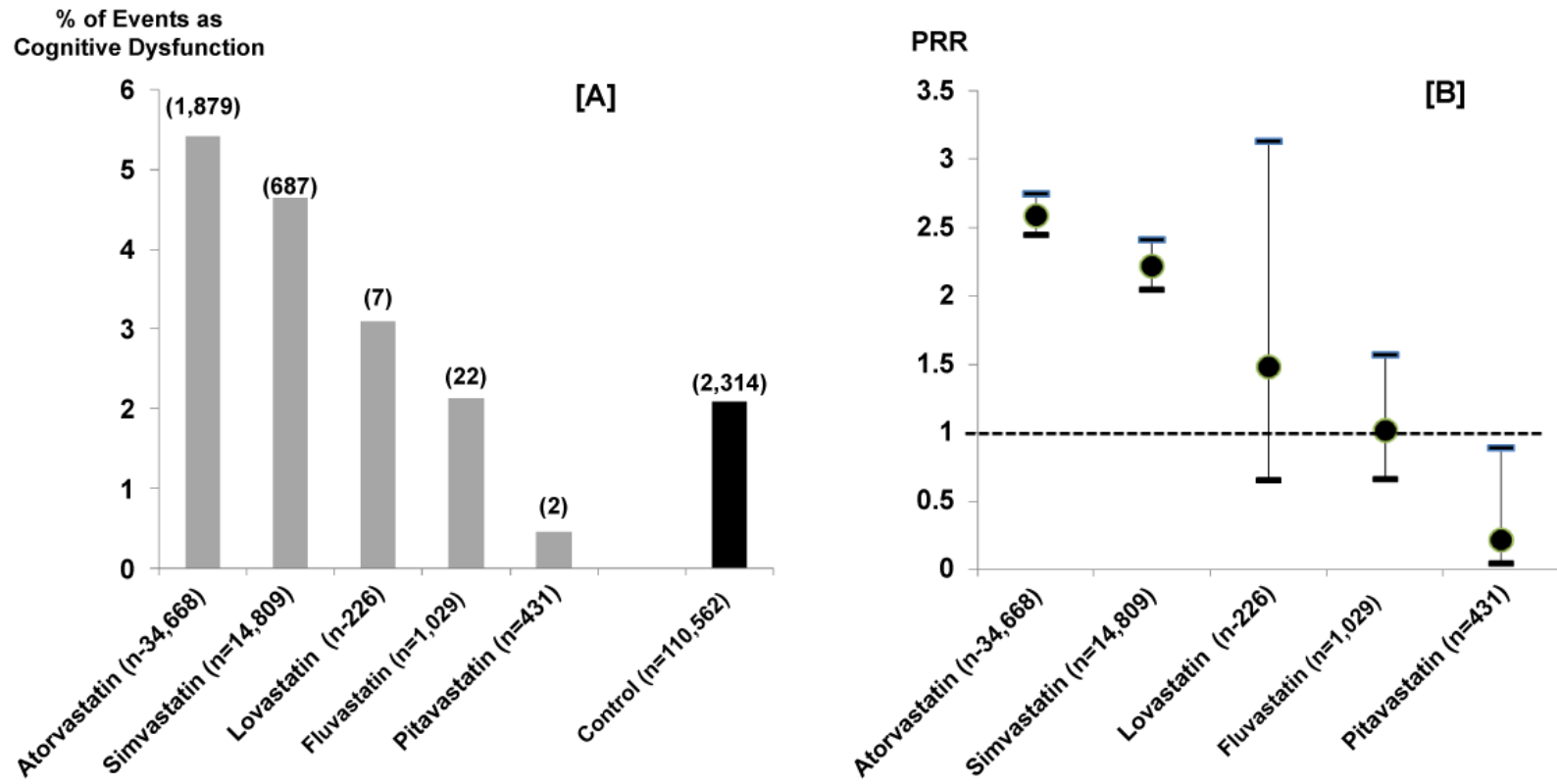

Figure 1: Proportional Reporting Ratios (PRR) of Individual Lipophilic Statins to Control Drugs for Cognitive Dysfunction. Panel A: Percentage of all adverse events listed as cognitive dysfunction by type of statin. The numbers in parentheses represent cognitive dysfunction adverse event reports. Panel B: Proportional reporting ratios (PRR) comparing individual statins to the control drugs. The filled circles represent the PRR point estimate; the horizontal lines represent the lower and upper $95 \%$ confidence limits.

\section{Subgroup analyses}

For the two statins with the highest signal (atorvastatin and simvastatin) for adverse cognitive outcomes (amnesia and cognitive disorder), subgroup analyses were conducted. For atorvastatin, the PRR versus all control drugs for amnesia was markedly higher for reports that were submitted by health professionals ( $P R R=11.84,95 \%$ confidence interval: $9.60,14.62)$ compared to reports submitted by consumers ( $\mathrm{PRR}=3.77,95 \%$ confidence interval: 3.17, 4.49). For reports of atorvastatin and the occurrence of cognitive disorder, the PRRs were exceptionally high $(>12)$ among males and during the time period 2006-2009. For simvastatin adverse reports, PRRs were generally similar among all subgroups except an apparent higher proportion of reports for cognitive disorder during the period 2010-2011.

\section{Discussion}

In this analysis of the FDA AERS database, we identified a significantly higher proportion of adverse reports of cognitive dysfunction associated with commonly prescribed potent, lipophilic statins, specifically, atorvastatin and simvastatin, compared to less frequently prescribed lipophilic statins (fluvastatin, lovastatin, pitavastatin) and to selected drugs commonly used in the general population. In addition, hydrophilic statins (rosuvastatin and pravastatin) showed essentially no evidence suggestive of heightened risk of cognitive dysfunction and were shown to have a statistically lower proportion of reported cognitive dysfunction compared to hydrochlorothiazide, a diuretic commonly prescribed for blood pressure management. Lipophilic statins metabolized through the CYP2C9 pathway (pitavastatin and fluvastatin; Table 1) or with combined low absorption and bioavailability (lovastatin) were associated with relatively few adverse reports in the AERS database with similar reports (proportionally) of cognitive dysfunction as the control drugs in aggregate.

\section{Potential adverse mechanism}

Statins can be classified as having greater lipophilic or hydrophilic solubility properties, with lipophilic statins tending to achieve higher levels of exposure in non-hepatic tissues because lipophilic statins passively and non-selectively diffuse into both hepatocyte and nonhepatocyte tissue; hydrophilic statins rely largely on active transport into hepatocyte to exert their effects $[6,33,35,37,49,50]$. High hepatoselectivity is thought to translate into a reduced risk of adverse effects [31,33]. Atorvastatin and simvastatin are potent lipid lowering statins, both metabolized through the CYP3A4 pathway, and possessing either long half-life (atorvastatin) or high absorption (simvastatin) [Table 1]. In contrast, fluvastatin undergoes extensive first-past metabolism rendering lower bioavailibilty and extensive protein binding reducing systemic exposure to non-hepatocyte tissue $[31,33]$. To maintain cell integrity and function, neurons require a sufficient supply of unesterified cholesterol. While all statins may potentially affect brain cholesterol metabolism by lowering available plasma cholesterol levels, lipophilic statins are thought to directly influence brain cholesterol metabolism by crossing the blood brain 
Page 6 of 9

barrier and inhibiting the synthesis of cholesterol within nerve cells or by limiting available extracellular cholesterol. This mechanism is consistent with reports of lipophilic statins having a greater distribution and severity of cognitive dysfunction, including memory loss, compared with hydrophilic statins [16,29,31,32,34,51].

Although the mechanism for transient or reversible cognitive dysfunction associated with statins is not known, Engelberg [52] proposed that adverse psychological and cognitive effects result when low cholesterol in brain cell membranes lead to lower lipid microviscosity, which in turn, may affect neurotransmitter exposure resulting in decreased synaptic binding and uptake. This effect may become intensified when lower cholesterol levels are achieved through the use of lipophilic statins, which may also adversely affect neuronal function though immunomodulatory or inflammatory mechanisms [53]. Statins are also known to lower circulating fat-soluble micronutrients including vitamin $\mathrm{E}$ which may indirectly affect the integrity of neuronal membranes resulting in neurocognitive deficits [21]. Our results lend support for the proposed mechanism whereby lipophilic statins with certain pharmacokinetic properties may differentially impact brain cholesterol synthesis and contribute to neuronal dysfunction in susceptible individuals. As statins are increasingly prescribed to a broader spectrum of at risk patients, insights as to the effects of highly lipophilic statins on brain metabolism are clinically relevant to assessing overall risk/benefit ratio and monitoring for potential adverse effects.

We did not observe a signal of adverse cognitive effects for the lipophilic statins pitavastatin, lovastatin, and fluvastatin. In addition to these statins having much lower historical market penetration compared to atorvastatin and simvastatin, they differ in pharmacokinetic properties including being metabolized primarily through the CYP2C9 pathway with relatively high absorption (pitavastatin and fluvastatin), or having a relatively short half-life (lovastatin). At this time, it is unclear as to the specific pharmacokinetics of atorvastatin and simvastatin in addition to being highly lipophilic that may predispose to adverse cognitive effects.

\begin{tabular}{|c|c|c|c|c|c|c|c|c|c|}
\hline \multirow[t]{2}{*}{ Cognitive Adverse Event } & \multicolumn{3}{|l|}{ Control } & \multicolumn{3}{|c|}{ Atorvastatin } & \multirow[t]{2}{*}{ PRR } & \multirow[t]{2}{*}{95 C.I. } & \multirow[t]{2}{*}{$P$-Value } \\
\hline & $\mathbf{N}$ & Events & $\%$ & $\mathbf{N}$ & Events & $\%$ & & & \\
\hline Amnesia & 110,562 & 433 & 0.39 & 34,668 & 1,102 & 3.17 & 8.12 & $7.26,9.08$ & $<0.0001$ \\
\hline Cognitive disorder & 110,562 & 93 & 0.08 & 34,668 & 293 & 0.85 & 10.05 & $7.91,12.77$ & $<0.0001$ \\
\hline Confusion state & 110,562 & 1,110 & 1.10 & 34,668 & 337 & 0.97 & 0.97 & $0.86,1.10$ & 0.63 \\
\hline Dementia & 110,562 & 377 & 0.34 & 34,668 & 199 & 0.57 & 1.68 & $1.41,2.01$ & $<0.0001$ \\
\hline Memory impairment & 110,562 & 480 & 0.43 & 34,668 & 440 & 1.27 & 2.92 & $2.56,3.33$ & $<0.0001$ \\
\hline Delusion & 110,562 & 63 & 0.08 & 34,668 & 7 & 0.02 & 0.35 & $0.15,0.80$ & 0.005 \\
\hline \multirow[t]{3}{*}{ Any Cognitive Event } & 110,562 & 2,314 & 2.09 & 34,668 & 1,879 & 5.42 & 2.59 & $2.44,2.75$ & $<0.0001$ \\
\hline & \multicolumn{3}{|l|}{ Control } & \multicolumn{3}{|c|}{ Simvastatin } & PRR & 95 C.I. & $P$-Value \\
\hline & $\mathbf{N}$ & Events & $\%$ & $\mathbf{N}$ & Events & $\%$ & & & \\
\hline Amnesia & 110,562 & 433 & 0.39 & 14,138 & 331 & 2.24 & 5.98 & $5.17,6.91$ & $<0.0001$ \\
\hline Cognitive disorder & 110,562 & 93 & 0.08 & 14,138 & 75 & 0.51 & 6.31 & $4.60,8.64$ & $<0.0001$ \\
\hline Confusion state & 110,562 & 1,110 & 1.10 & 14,138 & 196 & 1.32 & 1.38 & $1.18,1.61$ & $<0.0001$ \\
\hline Dementia & 110,562 & 377 & 0.34 & 14,138 & 44 & 0.30 & 0.91 & $0.66,1.26$ & 0.63 \\
\hline Memory impairment & 110,562 & 480 & 0.43 & 14,138 & 142 & 0.96 & 2.32 & $1.91,2.81$ & $<0.0001$ \\
\hline Delusion & 110,562 & 63 & 0.08 & 14,138 & 12 & 0.08 & 1.49 & $0.76,2.84$ & 0.28 \\
\hline Any Cognitive Event & 110,562 & 2,314 & 2.09 & 14,138 & 687 & 4.64 & 2.22 & $2.04,2.31$ & $<0.0001$ \\
\hline
\end{tabular}

Table 3: Proportional Reporting Ratios (PRR) of Individual Cognitive Adverse Events Comparing Atorvastatin and Simvastatin to Control Drugs.

\section{Passive surveillance of adverse events}

The AERS is a voluntary reporting system (passive surveillance). In our analysis, statin-induced adverse cognitive effects were significantly more likely to be reported to the FDA AERS database by consumers, both men and women, than by physicians or other health care providers. Warnings issued by the FDA are relevant to the practice community and consumers, yet the propensity to report adverse events may differ. From a survey of 650 community-based statin users studied to explore patient-physician interactions related to patient reported adverse effects of statins, Golomb and colleagues [22] found that of 113 respondents who reported adverse cognitive symptoms while on statins, only $65 \%$ reported their symptoms to their physician. Of these, nearly $50 \%$ of respondents reported that their physician denied any relationship between their symptoms and statin use. An additional $34 \%$ of respondents received no confirmation from their 
physician that their symptoms were, or were not, related to statin use. Golomb and coauthors [22] concluded that physicians may be missing less well-known adverse events such that post-marketing surveillance supported by patient-targeted reporting of adverse events may represent an important supplement to clinician reported adverse events, particularly for new or under-recognized symptoms.

In addition, it is noteworthy that voluntary reporting to AERS captures only a fraction of all true adverse events, estimated at between $1 \%$ to $10 \%$ [41,54]. In our analysis, a total of 2,566 reports of cognitive dysfunction were present for atorvastatin and simvastatin in AERS [Table 3] over an approximate 8-year period. By extrapolation, and assuming a true causal relationship between these two statins and cognitive dysfunction, this translates to roughly 3,000 to 30,000 occurrences each year. The magnitude of these crude estimates provides a rationale for greater awareness and surveillance of potential statin-induced adverse cognitive effects. While clinicians recognize that all drugs have the potential to exert adverse effects, the occurrence of cognitive dysfunction, including memory impairment, cognitive disorder, and dementia represent particularly worrisome adverse effects that may significantly impact quality of life and thus impact treatment options and medication adherence.

To date, clinician awareness of the potential of statins to induce memory impairment and symptoms of dementia may be underrecognized as compared to the more widely recognized effects of statins on muscle tissue. Complicating the recognition of the potential detrimental cognitive effects of statins is paradoxical experimental $[23,25,55]$ and clinical research $[56,58,59]$ indicating significant benefits for statins in reducing the neuropathological features and severity of cognitive symptoms in Alzheimer's and vascular dementia. In many of these studies, investigators chose to test their hypotheses using atorvastatin, simvastatin and lovastatin based on the suspected pleiotropic and lipophilic properties. Thus, clinicians may be preemptively prescribing highly lipophilic statins for cognitive protection in high-risk patients.

\section{Treatment selection}

In 2009, 211 million prescriptions for statins were recorded which exceeds the estimated prevalence of cardiovascular, coronary artery and cerebrovascular disease combined $[60,61]$ and reflects the extent to which statins are widely prescribed for primary prevention. In the clinical setting, the selection of a particular statin has been traditionally based on formulary constraints and the potency of a given statin to achieve targeted lipid lowering goals for individual patients. As there are no current recommendations for the selection of statins based on solubility properties or adverse event potential, our analysis provides emerging support for the clinical utility of matching individualized patient risk assessments with the relative solubility properties of the statin to reduce the likelihood of more severe cognitive adverse effects. A greater awareness of the effects of lipophilic versus hydrophilic statins may allow informed substitutions of better tolerated statins, thereby reducing adverse events and improving patient safety, adherence, and outcomes. This is consistent with a recent literature review that suggested that less lipophilic statins may be a logical alternative in cases where cognitive impairment that is secondary to a more lipophilic statin is suspected [32]. However, this review tended to minimize the risk of cognitive impairment with statin use by concluding it to be a rare occurrence, a conclusion at odds with the sheer number of adverse reports in the AERS database and known underestimation of true cases with this database. Going forward, epidemiological and prospective clinical research is needed to guide clinical decision-making in statin selection.

In addition, clinical decision making for statin use for the purpose of primary versus secondary prevention of cardiovascular disease has blurred over time. A Cochrane review [19] reported only limited evidence that primary prevention with statins may be cost effective and improve patient quality of life, and urged caution in prescribing statins for primary prevention among persons at low cardiovascular risk. Taking into account conventionally-recognized potential side effects of statins, absence of compelling primary prevention data in both children and adults (i.e. randomized controlled trials), and our results suggesting a greater occurrence of statin-induced cognitive dysfunction in adults (atorvastatin and simvastatin), we echo the need for great caution in the selection and use of statin therapy for children overall and adults at low cardiovascular risk.

\section{Limitations}

There are limitations to the FDA AERS database. AERS provides a tool for signal detection through the use of pharmacovigilance, which intends to search for unidentified patterns and automatically detect important signals [44]. A signal warrants further investigation but is not an "alert" which mandates wider communication or regulatory change. Statistical data mining tools, such as the PRR, allow the detection of signals that is not always possible using case series or reviews. The information from these types of studies identifies areas which are appropriate for additional study as well as continued surveillance. Causality assessments cannot be performed using the AERS database due to underreporting of events, variable reporting quality, reporting biases, and substantial missing data [62]. Moreover, numerator and denominator data are not available to provide incidence or risk estimates, and differences in magnitude of signal scores do not directly imply differences in risk [62].

\section{Conclusions}

To our knowledge, this is the first analysis of the AERS database to distinguish differential associations of lipophilic and hydrophilic statins on reported cognitive dysfunction. Our analysis identified potent, lipophilic statins (atorvastatin and simvastatin) as receiving a significantly greater proportion of reported adverse neurocognitive effects compared to other less potent or less lipophilic statins or hydrophilic solubility properties. These findings provide direction for future epidemiological and clinical research, as well as insights for informed treatment options, substitutions of more tolerable statins, and improved patient safety and adherence.

\section{Acknowledgements}

The contents do not represent the views of the United States Food and Drug Administration, Department of Veterans Affairs, or the United States Government. This work was completed through unfunded effort by the authors.

\section{Conflict of Interest Statement}

Dr. Sahebzamani takes full responsibility for the conduct of this analysis and results and conclusions presented. All authors have contributed materially to this manuscript and approve the final draft submitted. None of the authors has a conflict of interest with this work. 
Author contributions are as follows: Frances M. SahebzamaniConception, initiation, analysis, and writing (no conflict); Cindy L. Munro-Conception, critical review, and revisions (no conflict); Oscar C. Marroquin-Critical review and revisions (no conflict); David M. Diamond-Critical review and revisions (no conflict); Erin KellerCritical review and revisions (no conflict); Kevin E. Kip-Conception, initiation, analysis, and writing (no conflict).

\section{References}

1. Healy M (2010) New articles highlight rift over statins. Los Angeles Times, Los Angeles.

2. Stone NJ, Robinson J, Lichtenstein AH, Merz CN, Blum CB, et al. (2013) ACC/AHA Guideline on the Treatment of Blood Cholesterol to Reduce Atherosclerotic Cardiovascular Risk in Adults: A Report of the American College of Cardiology/American Heart Association Task Force on Practice Guidelines. J Am Coll Cardiol 63: 2889-2934.

3. Ioannidis JP (2014) More than a billion people taking statins?: Potential implications of the new cardiovascular guidelines. JAMA 311: 463-464.

4. Wassmann S, Laufs U, Bäumer AT, Müller K, Ahlbory K, et al. (2001) HMG-CoA reductase inhibitors improve endothelial dysfunction in normocholesterolemic hypertension via reduced production of reactive oxygen species. Hypertension 37: 1450-1457.

5. Shitara Y, Sugiyama Y (2006) Pharmacokinetic and pharmacodynamic alterations of 3-hydroxy-3methylglutaryl coenzyme A (HMG-CoA) reductase inhibitors: drug-drug interactions and interindividual differences in transporter and metabolic enzymes. Pharmacol Ther 112: 71-105.

6. Kim MC, Ahn Y, Jang SY, Cho KH, Hwang SH, et al. (2011) Comparison of clinical outcomes of hydrophilic and lipophilic statins in patients with acute myocardial infarction. Korean J Intern Med 26: 294-303.

7. Law MR, Wald NJ, Rudnicka AR (2003) Quantifying effect of statins on low density lipoprotein cholesterol, ischaemic heart disease and stroke. systematic review and meta-analysis, British Medical Journal 326: 1-7.

8. Guthrie RM (2006) Rising to the challenge of treating high-risk patients. Am J Manag Care 12: S318-324.

9. Barter P, Gotto AM, LaRosa JC, Maroni J, Szarek M, et al. (2007) HDL cholesterol, very low levels of LDL cholesterol, and cardiovascular events. N Engl J Med 357: 1301-1310.

10. Law MR, Thompson SG, Wald NJ (1994) Assessing possible hazards of reducing serum cholesterol. BMJ 308: 373-379.

11. Kaplan JR, Muldoon MF, Manuck SB, Mann JJ (1997) Assessing the observed relationship between low cholesterol and violence-related mortality. Implications for suicide risk. Ann N Y Acad Sci 836: 57-80.

12. Muldoon MF, Barger SD, Ryan CM, Flory JD, Lehoczky JP, et al. (2000) Effects of lovastatin on cognitive function and psychological well-being. Am J Med 108: 538-546.

13. Muldoon MF, Manuck SB, Mendelsohn AB, Kaplan JR, Belle SH (2001) Cholesterol reduction and non-illness mortality: meta-analysis of randomised clinical trials. BMJ 322: 11-15.

14. Dale KM, Coleman CI, Henyan NN, Kluger J, White CM (2006) Statins and cancer risk: a meta-analysis. JAMA 295: 74-80.

15. Alsheikh-Ali AA, Maddukuri PV, Han H, Karas RH (2007) Effect of the magnitude of lipid lowering on risk of elevated liver enzymes, rhabdomyolysis, and cancer: insights from large randomized statin trials. J Am Coll Cardiol 50: 409-418.

16. Golomb BA, Evans MA, Dimsdale JE, White HL (2012) Effects of statins on energy and fatigue with exertion: results from a randomized controlled trial. Arch Intern Med 172: 1180-1182.

17. de Lorgeril M, Salen P, Abramson J, Dodin S, Hamazaki T, et al. (2010) Cholesterol lowering, cardiovascular diseases, and the rosuvastatinJUPITER controversy: a critical reappraisal. Arch Intern Med 170: 1032-1036.
18. Ebrahim S (2011) Multiple risk factor interventions for primary prevention of coronary heart disease. Cochrane Database Syst Rev 4: CD001561.

19. Taylor F, Huffman MD, Macedo AF, Moore TH, Burke M, et al. (2013) Statins for the primary prevention of cardiovascular disease. Cochrane Database Syst Rev 1: CD004816.

20. Cholesterol Treatment Trialists' (CTT) Collaborators, Mihaylova B, Emberson J, Blackwell L, Keech A, et al. (2012) The effects of lowering LDL cholesterol with statin therapy in people at low risk of vascular disease: meta-analysis of individual data from 27 randomised trials. Lancet 380: 581-590.

21. McGuinness B, O'Hare J, Craig D, Bullock R, Malouf R, et al. (2010) Statins for the treatment of dementia. Cochrane Database Syst Rev CD007514.

22. Golomb BA, McGraw JJ, Evans MA, Dimsdale JE (2007) Physician response to patient reports of adverse drug effects: implications for patient-targeted adverse effect surveillance. Drug Saf 30: 669-675.

23. Wolozin B, Kellman W, Ruosseau P, Celesia GG, Siegel G (2000) Decreased prevalence of Alzheimer disease associated with 3-hydroxy-3methyglutaryl coenzyme A reductase inhibitors. Arch Neurol 57: 1439-1443.

24. Li G, Larson EB, Sonnen JA, Shofer JB, Petrie EC, et al. (2007) Statin therapy is associated with reduced neuropathologic changes of Alzheimer disease. Neurology 69: 878-885.

25. Kurata T, Miyazaki K, Kozuki M, Panin VL, Morimoto N, et al. (2011) Atorvastatin and pitavastatin improve cognitive function and reduce senile plaque and phosphorylated tau in aged APP mice. Brain Res 1371: 161-170.

26. Vance JE (2012) Dysregulation of cholesterol balance in the brain: contribution to neurodegenerative diseases. Dis Model Mech 5: 746-755.

27. Corrao G, Ibrahim B, Nicotra F, Zambon A, Merlino L, et al. (2013) Long-term use of statins reduces the risk of hospitalization for dementia. Atherosclerosis 230: 171-176.

28. Administration (2012) FDA announces safety changes in labeling for some cholesterol-lowering drugs. U.S Food and Drug Administration.

29. Thelen KM, Rentsch KM, Gutteck U, Heverin M, Olin M, et al. (2006) Brain cholesterol synthesis in mice is affected by high dose of simvastatin but not of pravastatin. J Pharmacol Exp Ther 316: 1146-1152.

30. Ancelin ML, Carrière I, Barberger-Gateau P, Auriacombe S, Rouaud O, et al. (2012) Lipid lowering agents, cognitive decline, and dementia: the three-city study. J Alzheimers Dis 30: 629-637.

31. Sparks DL, Connor DJ, Browne PJ, Lopez JE, Sabbagh MN (2002) HMGCoA reductase inhibitors (statins) in the treatment of Alzheimer's disease and why it would be ill-advise to use one that crosses the blood-brain barrier. J Nutr Health Aging 6: 324-331.

32. Rojas-Fernandez CH, Cameron JC (2012) Is statin-associated cognitive impairment clinically relevant? A narrative review and clinical recommendations. Ann Pharmacother 46: 549-557.

33. Hamelin BA, Turgeon J (1998) Hydrophilicity/lipophilicity: relevance for the pharmacology and clinical effects of HMG-CoA reductase inhibitors. Trends Pharmacol Sci 19: 26-37.

34. Locatelli S, Lütjohann D, Schmidt HH, Otto C, Beisiegel U, et al. (2002) Reduction of plasma 24S-hydroxycholesterol (cerebrosterol) levels using high-dosage simvastatin in patients with hypercholesterolemia: evidence that simvastatin affects cholesterol metabolism in the human brain. Arch Neurol 59: 213-216.

35. Schachter M (2005) Chemical, pharmacokinetic and pharmacodynamic properties of statins: an update. Fundam Clin Pharmacol 19: 117-125.

36. Heverin M, Meaney S, Lütjohann D, Diczfalusy U, Wahren J, et al. (2005) Crossing the barrier: net flux of 27-hydroxycholesterol into the human brain. J Lipid Res 46: 1047-1052.

37. Pfefferkorn JA, Song Y, Sun KL, Miller SR, Trivedi BK, et al. (2007) Design and synthesis of hepatoselective, pyrrole-based HMG-CoA reductase inhibitors. Bioorg Med Chem Lett 17: 4538-4544. 
Citation: Sahebzamani FM, Munro CL, Marroquin OC, Diamond DM, Keller E, et al. (2014) Examination of the FDA Warning for Statins and Cognitive Dysfunction. J Pharmacovigilance 2: 141. doi:10.4172/2329-6887.1000141

Page 9 of 9

38. CibiäkovÃ $\tilde{A}_{i}$ L (2011) Statins and their influence on brain cholesterol. J Clin Lipidol 5: 373-379.

39. Casey C, Vellozzi C, Mootrey GT, Chapman LE, McCauley M, et al. (2006) Surveillance guidelines for smallpox vaccine (vaccinia) adverse reactions. MMWR Recomm Rep 55: 1-16.

40. Medical Dictionary for Regulatory Activities.

41. Kessler DA (1993) Introducing MEDWatch. A new approach to reporting medication and device adverse effects and product problems. JAMA 269: 2765-2768.

42. Nebeker JR, Barach P, Samore MH (2004) Clarifying adverse drug events: a clinician's guide to terminology, documentation, and reporting. Ann Intern Med 140: 795-801.

43. Bate A, Evans SJ (2009) Quantitative signal detection using spontaneous ADR reporting. Pharmacoepidemiol Drug Saf 18: 427-436.

44. Sakaeda T, Kadoyama K, Okuno Y (2011) Adverse event profiles of platinum agents: data mining of the public version of the FDA adverse event reporting system, AERS, and reproducibility of clinical observations. Int J Med Sci 8: 487-491.

45. Mukhtar RY, Reid J, Reckless JP (2005) Pitavastatin. Int J Clin Pract 59: 239-252.

46. Moghadasian MH (1999) Clinical pharmacology of 3-hydroxy-3methylglutaryl coenzyme A reductase inhibitors. Life Sci 65: 1329-1337.

47. Biondi E (2011) Prescription of lipophilic statins to Alzheimer's disease patients: some controversies to consider. Neurol Sci 32: 195-201.

48. Evans SJ, Waller PC, Davis S (2001) Use of proportional reporting ratios (PRRs) for signal generation from spontaneous adverse drug reaction reports. Pharmacoepidemiol Drug Saf 10: 483-486.

49. Corsini A, Bellosta S, Baetta R, Fumagalli R, Paoletti R, et al. (1999) New insights into the pharmacodynamic and pharmacokinetic properties of statins. Pharmacol Ther 84: 413-428.

50. White CM (2002) A review of the pharmacologic and pharmacokinetic aspects of rosuvastatin. J Clin Pharmacol 42: 963-970.

51. Wagstaff LR, Mitton MW, Arvik BM, Doraiswamy PM (2003) Statinassociated memory loss: analysis of 60 case reports and review of the literature. Pharmacotherapy 23: 871-880.
52. Engelberg H (1992) Low serum cholesterol and suicide. Lancet 339: 727-729.

53. Chauhan NB, Siegel GJ, Feinstein DL (2004) Effects of lovastatin and pravastatin on amyloid processing and inflammatory response in TgCRND8 brain. Neurochem Res 29: 1897-1911.

54. Nestle FO, Conrad C, Tun-Kyi A, Homey B, Gombert M, et al. (2005) Plasmacytoid predendritic cells initiate psoriasis through interferonalpha production. J Exp Med 202: 135-143.

55. Fassbender K, Simons M, Bergmann C, Stroick M, Lutjohann D, et al. (2001) Simvastatin strongly reduces levels of Alzheimer's disease beta amyloid peptides Abeta 42 and Abeta 40 in vitro and in vivo. Proc Natl Acad Sci U S A 98: 5856-5861.

56. Jick H, Zornberg GL, Jick SS, Seshadri S, Drachman DA (2000) Statins and the risk of dementia. Lancet 356: 1627-1631.

57. Sparks DL, Sabbagh MN, Connor DJ, Lopez J, Launer LJ, et al. (2005) Atorvastatin for the treatment of mild to moderate Alzheimer disease: preliminary results. Arch Neurol 62: 753-757.

58. Sparks DL, Connor DJ, Sabbagh MN, Petersen RB, Lopez J, et al. (2006) Circulating cholesterol levels, apolipoprotein E genotype and dementia severity influence the benefit of atorvastatin treatment in Alzheimer's disease: results of the Alzheimer's Disease Cholesterol-Lowering Treatment (ADCLT) trial. Acta Neurol Scand Suppl 185: 3-7.

59. Sparks DL, Kryscio RJ, Sabbagh MN, Connor DJ, Sparks LM, et al. (2008) Reduced Risk of Incident AD with Elective Statin use in a clinical trial cohort. Curr Alzheimer Res 5: 416-421.

60. IMS Institute for Healthcare Informatics (2012) The use of medicines in the United States: Review of 1-46.

61. Roger VL, Go AS, Lloyd-Jones DM, Adams RJ, Berry JD, et al. (2011) Heart disease and stroke statistics--2011 update: a report from the American Heart Association. Circulation 123: e18-18e209.

62. Jones SC, Sorbello A, Boucher RM (2011) Fluoroquinolone-associated myasthenia gravis exacerbation: evaluation of postmarketing reports from the US FDA adverse event reporting system and a literature review. Drug Saf 34: 839-847. 